ISSN 0103-9954

\title{
INFLUÊNCIA DO ÂNGULO ENTRE O ESFORÇO APLICADO E A DIREÇÃO DAS FIBRAS DA MADEIRA SOBRE A RESISTÊNCIA AO CISALHAMENTO
}

\author{
INFLUENCE OF THE ANGLE BETWEEN APPLIED FORCE AND WOOD GRAIN DIRECTION ON \\ SHEAR STRENGTH
}

\author{
Norman Barros Logsdon ${ }^{1}$ Zenesio Finger ${ }^{2}$ José Manoel Henriques de Jesus ${ }^{3}$
}

\section{RESUMO}

A madeira é um material anisotrópico e suas características de resistência variam com o ângulo entre o esforço e a direção das fibras $(\alpha)$. A norma brasileira NBR 7190 - Projeto de Estruturas de Madeira prevê a correção da resistência às tensões normais, sempre que $\alpha>6^{\circ}$, e recomenda aplicar a expressão de Hankinson aos valores de cálculo. Entretanto, a referida norma é omissa sobre esta correção às tensões tangenciais. Além disso, não há comprovação experimental sobre a validade de utilizar esta expressão em valores de cálculo, oriundos de valores característicos. Foram objetivos deste trabalho: pesquisar na bibliografia outras expressões utilizadas; verificar a validade destas expressões para representar a variação das resistências ao cisalhamento com o ângulo $\alpha$ e verificar a validade de aplicação destas expressões em valores característicos. Para isto, utilizou-se uma amostra formada por doze pranchas de madeira. De cada prancha foram retirados corpos de prova de cisalhamento, com diferentes inclinações das fibras, que foram ensaiados em acordo com a atual NBR 7190, fornecendo as resistências ao cisalhamento, para diferentes $\alpha$. Quatro modelos (Hankinson, Senos, Karlsen e Keylwerth) foram avaliados, utilizando-se os testes de pairing e dos desvios. O modelo que melhor representou o fenômeno, nos dois casos, foi o de Keylwerth. Palavras-chave: resistência ao cisalhamento; resistência característica; inclinação das fibras.

\begin{abstract}
Wood is an anisotropic material and its resistance characteristics vary with the angle between the effort and the fiber direction $(\alpha)$. The Brazilian Code NBR 7190 - Design of Wooden Structures, foresees the strength correction to the normal stress, whenever $\alpha>6^{\circ}$, and it recommends to apply Hankinson's expression to design values. However, this referred code is omitted about this correction to the tangential stress. Besides, there is not experimental confirmation about the validity of using this expression on design values, originating from characteristic values. The objectives of this work were: to search in the bibliography other used expressions; to verify the validity of these expressions, to represent the variation of the shear strength, with the $\alpha$ angle; and, to verify the validity of application of these expressions in characteristic values. For this, a sample formed by twelve boards was used. From each board, specimens to shear tests were retired, with different fibers inclinations, which were tested in agreement with current NBR 7190, supplying the shear strength, for different $\alpha$ values. Four models (Hankinson, Sin, Karlsen and Keylwerth) were evaluated, using the pairing and deviation tests. The model that best represented the phenomenon, in both cases, was Keylwerth's.
\end{abstract}

Keywords: shear strength; characteristic strength; fibers inclination.

1 Engenheiro Civil, Dr., Professor Associado do Departamento de Engenharia Florestal, Faculdade de Engenharia Florestal, Universidade Federal de Mato Grosso, Av. Fernando Corrêa da Costa, s/n, CEP 78060-900, Cuiabá (MT), Brasil.logsdon@terra.com.br

2 Engenheiro Florestal, Dr., Professor Associado do Departamento de Engenharia Florestal, Faculdade de Engenharia Florestal, Universidade Federal de Mato Grosso, Av. Fernando Corrêa da Costa, s/n, CEP 78060-900, Cuiabá (MT), Brasil. fingerz@terra.com.br

3 Engenheiro Civil, Dr., Professor Associado do Departamento de Engenharia Civil, Faculdade de Arquitetura, Engenharia e Tecnologia, Universidade Federal de Mato Grosso, Av. Fernando Corrêa da Costa, s/n, CEP 78060-900, Cuiabá (MT), Brasil. jmhenriques@terra.com.br 


\section{INTRODUÇÃO}

A atual norma brasileira, NBR 7190 Projeto de Estruturas de Madeira, da Associação Brasileira de Normas Técnicas - ABNT (1997), prevê a correção da resistência de cálculo, para verificação de tensões normais inclinadas, sempre que o ângulo entre o esforço e a direção das fibras for superior a $6^{\circ}$, e recomenda aplicar a expressão de Hankinson, mas é omissa sobre esta correção para tensões tangenciais.

Devido ao seu arranjamento celular particular e à natureza de sua fisiologia, a madeira é um material anisotrópico. Por isto, suas propriedades mecânicas dependem da direção de aplicação do esforço em relação às suas fibras. Vários autores estudaram o assunto, alguns estabeleceram modelos teóricos, em geral considerando a madeira como um material ortotrópico, outros estabeleceram modelos empíricos, baseados em resultados de ensaios.

Entre os modelos empíricos, segundo Kollmann e Côté Jr. (1984), o modelo proposto por Hankinson, apresentado na Equação 1, com expoente $\mathrm{n}=2$, tem sido o mais utilizado para obter a resistência a uma tensão normal inclinada. Karlsen et al. (1967), por outro lado, recomendam o modelo empírico apresentado na Equação 2, com expoente $\mathrm{n}=3$.

Um estudo teórico, desenvolvido por Szücs (1992), concluiu sobre a possibilidade de utilizar para a correção de resistências, às tensões normais inclinadas, tanto por tração como por compressão, a Equação 3, com o expoente $\mathrm{n}=2$, já, segundo Pfeil (1978), a norma alemã DIN-1052 adotava esta expressão com o expoente $n=1$. Keylwerth, segundo Kollmann e Côté Jr. (1984), também desenvolveu uma expressão teórica, de expoente $n=2$, mas para a variação do módulo de elasticidade, que, para as resistências, assume a forma da Equação 4.

$$
\begin{gathered}
\mathrm{f}_{\alpha}=\frac{\mathrm{f}_{0} \cdot \mathrm{f}_{90}}{\mathrm{f}_{0} \cdot \operatorname{sen}^{\mathrm{n}} \alpha+\mathrm{f}_{90} \cdot \cos ^{\mathrm{n}} \alpha} \\
\mathrm{f}_{\alpha}=\frac{\mathrm{f}_{0}}{1+\left(\frac{\mathrm{f}_{0}}{\mathrm{f}_{90}}-1\right) \cdot \operatorname{sen}^{\mathrm{n}} \alpha} \\
\mathrm{f}_{\alpha}=\mathrm{f}_{0}-\left(\mathrm{f}_{0}-\mathrm{f}_{90}\right) \cdot \operatorname{sen}^{\mathrm{n}} \alpha
\end{gathered}
$$

$$
\mathrm{f}_{\alpha}=\frac{\mathrm{f}_{0}}{\left(\cos ^{\mathrm{n}} \alpha-\frac{\mathrm{f}_{0}}{\mathrm{f}_{90}} \operatorname{sen}^{\mathrm{n}} \alpha\right) \cdot \cos (2 . \alpha)+\frac{\mathrm{f}_{0}}{\mathrm{f}_{45}} \operatorname{sen}^{\mathrm{n}}(2 . \alpha)}
$$

\section{Em que:}

$\mathrm{f}_{\alpha}=$ Resistência a uma tensão normal inclinada de um ângulo $\alpha$ em relação às fibras da madeira;

$\mathrm{f}_{0}=$ Resistência a uma tensão normal paralela $\left(\alpha=0^{\circ}\right)$ em relação às fibras da madeira;

$\mathrm{f}_{45}=$ Resistência a uma tensão normal inclinada de um ângulo $\alpha=45^{\circ} \mathrm{em}$ relação às fibras da madeira; $\mathrm{f}_{90}=$ Resistência a uma tensão normal perpendicular $\left(\alpha=90^{\circ}\right)$ em relação às fibras da madeira;

$\alpha=$ Ângulo entre o esforço aplicado e a direção das fibras;

$\mathrm{n}=$ Expoente dos termos trigonométricos.

Também há controvérsia, entre os autores, sobre o valor de expoente dos termos trigonométricos (n) nas Equações 1 a 4. Alguns autores indicam coeficientes diferentes conforme o tipo de solicitação em análise, outros indicam coeficientes diferentes para diferentes teores de umidade da madeira.

O surgimento de novos materiais foi acompanhado do desenvolvimento de critérios mais amplos de resistência, entre eles a teoria geral de resistência elaborada por Tsai e Wu (1971) para materiais anisotrópicos. Este critério não será avaliado, neste trabalho, pelas seguintes razões: 1) Os parâmetros usados pelo critério não são corriqueiramente obtidos na caracterização físicomecânica de madeiras, o que tornaria necessário rever ou ampliar sobremaneira os ensaios utilizados com este fim; 2) O exagerado número de parâmetros utilizado pelo critério, praticamente obriga a admitir hipóteses simplificadoras, com o intuito de diminuir o número de parâmetros, que dificilmente serão verificadas. Além disso, a avaliação do critério de Tsai e Wu (1971), para tensões normais de tração, realizada por Nicolas et al. (2008), mostrou boa aproximação com a expressão de Hankinson (Equação 1), embora, tenha, para a inclinação de $15^{\circ}$ e na melhor situação analisada, superestimado a resistência em mais de 10\% (inaceitável em engenharia), enquanto a expressão de Hankinson, também para a inclinação de $15^{\circ}$ e na melhor situação analisada $(n=1,5)$, subestimou a resistência em torno de 5\%. Chama a atenção, neste estudo de Nicolas et al. (2008), o rápido crescimento do 
erro cometido ao se utilizar valores diferentes do expoente " $n$ ", afastando-se do valor ideal, na expressão de Hankinson, para a inclinação de $15^{\circ}$ foi superestimada a resistência em mais de $10 \%$, com $\mathrm{n}=2$, e em mais de $40 \%$, com $\mathrm{n}=2,5$.

Não se encontrou na literatura nenhum estudo a respeito da variação das tensões tangenciais com a inclinação das fibras, embora, Karlsen et al. (1967) apresentem uma figura, montada a partir da Equação 2, com $\mathrm{n}=3$, mostrando a variação das tensões admissíveis de cisalhamento com o ângulo entre o esforço aplicado e a direção das fibras.

Todos os estudos encontrados na bibliografia apontam para a variação em um indivíduo (ou para a média dos resultados obtidos em diferentes indivíduos), mas a aplicação em projetos de estruturas de madeira utiliza-se de valores de cálculo de resistências, que originam de valores característicos. Valores característicos, além da variação individual do efeito em questão, estão associados à variabilidade de resultados dentro de uma árvore e ao longo das árvores da espécie do lote avaliado. Desta forma, torna-se necessário avaliar como variam valores característicos de resistência - associados à parcela menos resistente da madeira quando se varia o ângulo entre o esforço aplicado e a direção das fibras.

Estas informações indicam que o estudo da variação da resistência com o ângulo entre o esforço e a direção das fibras não está concluído. Não existe consenso entre autores sobre qual a melhor expressão a ser utilizada e, nem mesmo sobre o valor do expoente em determinada expressão e não se tem garantia experimental de que uma relação estudada para valores de ruptura possa ser aplicada a valores característicos. Especificamente para tensões de cisalhamento o problema ainda não foi abordado.
O objetivo deste trabalho foi estudar experimentalmente a influência do ângulo entre o esforço aplicado e a direção das fibras sobre a resistência ao cisalhamento da madeira e, especialmente, sobre sua resistência característica ao cisalhamento.

\section{MATERIAL E MÉTODO}

Para estudar a variação da resistência ao cisalhamento, com o ângulo entre o esforço aplicado e a direção das fibras é necessário utilizar amostras com a menor variação possível.

Para estudar a variação da resistência de cálculo com o ângulo entre o esforço aplicado e a direção das fibras, por sua vez, é necessário considerar a variabilidade da madeira dentro de uma árvore e entre as árvores da espécie. A atual norma brasileira, NBR 7190 da ABNT (1997), define a amostragem para um lote homogêneo de até $12 \mathrm{~m}^{3}$ de madeira, uma amostra de 12 corpos de prova, para cada tipo de ensaio.

Assim, tomou-se um lote mínimo formado por três árvores. De cada árvore foram retiradas quatro pranchas diametrais, no plano radiallongitudinal, totalizando 12 pranchas. De cada prancha foram retirados os corpos de prova como se apresenta na Figura 1. Desta forma, cada inclinação $\left(0^{\circ}, 10^{\circ}, 20^{\circ}, 30^{\circ}, 40^{\circ}, 45^{\circ}, 50^{\circ}, 60^{\circ}, 70^{\circ}, 80^{\circ}\right.$ e $\left.90^{\circ}\right)$ contou com 12 corpos de prova, totalizando 132 corpos de prova de cisalhamento. O material não utilizado foi identificado e armazenado de modo a permitir refazer qualquer corpo de prova, cujo resultado de ensaio apresentasse anomalia.

Devido à facilidade de obtenção utilizouse a espécie Aspidosperma populifolium A. DC., conhecida por peroba-mica, uma vez que é uma das

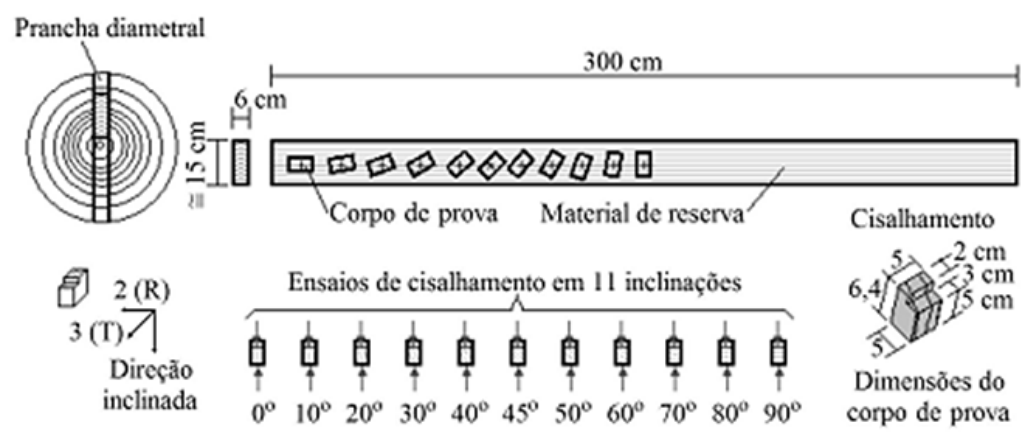

FIGURA 1: Plano de amostragem dos corpos de prova de uma prancha.

FIGURE 1: Sampling method to obtain specimens from a board. 
espécies em exploração no extremo norte do Estado de Mato Grosso. Todas as árvores tinham idade aproximada de 100 anos, diâmetro superior a 1,00 $\mathrm{m}$ e apenas madeira madura do cerne foi utilizada. O material foi coletado no Município de Cotriguaçu no Estado de Mato Grosso.

A fim de estabilizar a madeira a um teor de umidade próximo de $12 \%$, de modo a minimizar erros ao reportar os resultados dos ensaios ao teor de umidade de referência, foi providenciada uma secagem ao ar da madeira.

Inicialmente, a madeira foi desdobrada em peças, com seção de $6 \mathrm{~cm} \times 6 \mathrm{~cm}$, conforme o esquema da Figura 1. As peças foram identificadas e colocadas para secar ao ar até que a massa das peças de uma amostra de controle, composta por três peças (uma de cada árvore), apresentassem, constância de massa (variação inferior a $0,5 \%$ ) entre duas leituras sucessivas, no intervalo de um dia. Esta secagem prévia teve uma duração aproximada de 30 dias. Só após esta secagem prévia, as peças foram desdobradas em corpos de prova. Este procedimento minimizou a ocorrência de deformações, dos corpos de prova, por variação do teor de umidade.

Os ensaios de cisalhamento, dos quais foram obtidas as resistências ao cisalhamento, foram realizados em corpos de prova de dimensões $6,4 \mathrm{~cm} \times 5 \mathrm{~cm} \times 5 \mathrm{~cm}$ (com um pequeno degrau, de $1,4 \mathrm{~cm} \times 2 \mathrm{~cm} \times 5 \mathrm{~cm}$, para fixação do equipamento de ensaio, acarretando uma seção cisalhante de $5 \mathrm{~cm}$ x $5 \mathrm{~cm}$ ), com as direções das fibras bem definidas, utilizando a metodologia descrita na NBR 7190, da ABNT (1997), para o ensaio de cisalhamento paralelo às fibras. A massa de cada corpo de prova foi avaliada logo após o ensaio e após a secagem em estufa, a fim de obter a umidade no instante do ensaio, possibilitando reportar o resultado ao teor de umidade de referência de $12 \%$.

Assim, foi possível obter: a tensão de ruptura (Equação 5) e o teor de umidade dos corpos de prova no instante do ensaio (Equação 6).

$$
\begin{gathered}
\mathrm{f}_{\mathrm{v} \alpha}=\frac{\mathrm{F}_{\mathrm{v} \alpha, \max }}{\mathrm{A}_{\mathrm{v} \alpha}} \\
\mathrm{U}=\frac{\mathrm{m}_{\mathrm{u}}-\mathrm{m}_{0}}{\mathrm{~m}_{0}} \cdot 100 \%
\end{gathered}
$$

Em que:

$\mathrm{f}_{\mathrm{v} \alpha}=$ Resistência ao cisalhamento paralelo às fibras da madeira, em MPa;

$\mathrm{F}_{\mathrm{va} \text {,max }}=$ Máxima força cisalhante aplicada ao corpo de prova (carga de ruptura), em $\mathrm{N}$;

$\mathrm{A}_{\mathrm{v} \alpha}=$ Área da seção cisalhante do corpo de prova, num plano inclinado, de um ângulo " $\alpha$ " em relação às fibras, em $\mathrm{mm}^{2}$;

$\mathrm{U}=$ Teor de umidade do corpo de prova, no instante do ensaio, em \%;

$\mathrm{m}_{\mathrm{u}}=$ Massa do corpo de prova, no instante do ensaio, em g;

$\mathrm{m}_{0}=$ Massa do corpo de prova seco em estufa, em $\mathrm{g}$.

Para avaliação de massas foi utilizada uma balança analítica, com capacidade de 1,0-kg e sensibilidade de $0,01 \mathrm{~g}$. As dimensões foram avaliadas com um paquímetro digital, com capacidade de $10 \mathrm{~cm}$ e sensibilidade de $0,01 \mathrm{~mm}$. A secagem foi feita em estufa de esterilização e secagem que mantinha temperatura de $(103 \pm 2)^{\circ} \mathrm{C}$. A aplicação da carga foi feita em máquina universal de ensaios, com capacidade de $300 \mathrm{kN}$ e sensibilidade de $0,01 \mathrm{kN}$, a uma velocidade de carregamento de $2,5-\mathrm{MPa} / \mathrm{min}$.

Para reportar os resultados ao teor de umidade de referência foi utilizada a equação aferida por Logsdon (1998), apresentada na Equação 7, já com a notação adequada ao caso.

$$
\begin{gathered}
\mathrm{f}_{\mathrm{v} \alpha}=\frac{\mathrm{F}_{\mathrm{v} \alpha, \max }}{\mathrm{A}_{\mathrm{v} \alpha}} \\
\mathrm{U}=\frac{\mathrm{m}_{\mathrm{u}}-\mathrm{m}_{0}}{\mathrm{~m}_{0}} \cdot 100 \%
\end{gathered}
$$

Em que:

$\mathrm{f}_{\mathrm{v}, 12}=$ Resistência ao cisalhamento inclinado de um ângulo $\alpha$, em relação às fibras da madeira, ao teor de umidade de referência de $12 \%$;

$\mathrm{f}_{\mathrm{va}, \mathrm{U} \%}=$ Resistência ao cisalhamento inclinado de um ângulo $\alpha$, em relação às fibras da madeira, ao teor de umidade $\mathrm{U} \%$;

$\mathrm{U}=$ Teor de umidade da madeira no instante do ensaio, em \%.

Todos os modelos, apresentados na revisão bibliográfica, para representar a variação da resistência com o ângulo entre o esforço aplicado e a direção das fibras, são dependentes de, pelo menos, dois valores experimentais, a resistência na direção paralela às fibras $\left(\alpha=0^{\circ}\right)$ e na direção normal às fibras $\left(\alpha=90^{\circ}\right)$. Assim, uma eventual falha na avaliação destes valores, por má condução do ensaio ou pela 
existência de algum defeito localizado na madeira, que não foi percebido, comprometeria a avaliação do modelo. Para minimizar erros na avaliação dos modelos, decidiu-se refazer todo ensaio cujo resultado destoasse de seus pares.

Dos 12 corpos de prova com a mesma direção das fibras (um de cada prancha) foram obtidos os valores médios (Equação 8), incluindo o intervalo de confiança da média (Equação 9), e os valores característicos (Equação 10) como definidos na atual NBR 7190, da ABNT (1997).

$$
\begin{gathered}
\mathrm{x}_{\mathrm{wm}}=\frac{\sum_{\mathrm{i}=1}^{\mathrm{n}} \mathrm{x}_{\mathrm{i}}}{\mathrm{n}} \\
\overline{\mathrm{x}}-\mathrm{t}(\phi, 95 \%) \cdot \frac{\mathrm{s}_{\mathrm{x}}}{\sqrt{\mathrm{n}}} \leq \mu_{\mathrm{x}} \leq \overline{\mathrm{x}}+\mathrm{t}(\phi, 95 \%) \cdot \frac{\mathrm{s}_{\mathrm{x}}}{\sqrt{\mathrm{n}}} \\
\text { Máximo }\left\{\mathrm{x}_{1} ; 0,7 \mathrm{x}_{\mathrm{wm}} \text { e } \mathrm{x}_{\mathrm{wk}}=\left(2 \frac{\mathrm{x}_{1}+\mathrm{x}_{2}+\ldots+\mathrm{x}_{\frac{\mathrm{n}}{2}-1}}{\frac{\mathrm{n}}{2}-1}-\mathrm{x}_{\frac{\mathrm{n}}{2}}\right) 1,1\right\}
\end{gathered}
$$

Em que:

$\mathrm{x}_{\mathrm{i}}=$ Elemento "i" da variável " $\mathrm{x}$ ". Resistência, obtida no ensaio do corpo de prova " $i$ ". Os valores $x_{i}$ são ordenados crescentemente, de modo que $\mathrm{x}_{1}$ é a menor resistência obtida nos ensaios;

$\mathrm{x}_{\mathrm{wm}}=$ Estimativa da média da característica mecânica em questão, o mesmo que $\bar{x}$. Valor médio das resistências obtidas nos ensaios;

$\mathrm{n}=$ Número de corpos de prova ensaiados (tamanho da amostra);

$\mathrm{x}=$ Característica mecânica em questão (variável estudada);

$\bar{x}=$ Estimativa da média da característica mecânica em questão;

$\mathrm{s}_{\mathrm{x}}=$ Desvio padrão da amostra;

$\frac{\mathrm{s}_{\mathrm{x}}}{\sqrt{\mathrm{n}}}=$ Erro padrão de estimativa;

$\varphi=$ Número de graus de liberdade;

$\mathrm{t}(\varphi, 95 \%)=$ Valor do estatístico $\mathrm{t}$, para $\varphi$ graus de liberdade e $95 \%$ de probabilidade;

$\mu_{\mathrm{x}}=$ Média da característica mecânica em questão;

$\mathrm{x}_{\mathrm{wk}}=$ Operador para o valor característico da resistência.

Para avaliação dos modelos foram utilizados dois instrumentos estatísticos: o teste de pairing, que avalia se os desvios, entre os resultados de ensaio e os obtidos pelo modelo, têm média nula; e o teste dos desvios, que avalia se estes desvios não apresentam tendenciosidade ao longo do domínio.

Para avaliar a variação das resistências (tensões de ruptura) com o ângulo entre o esforço aplicado e a direção das fibras da madeira, optouse por estudar o comportamento dos resultados obtidos em cada prancha, já corrigidos para o teor de umidade de referência de $12 \%$, e, em seguida, verificar a existência de um comportamento comum às 12 pranchas.

Para avaliar a variação das resistências de cálculo com o ângulo entre o esforço aplicado e a direção das fibras, optou-se por estudar o comportamento das variáveis que compõem o modelo da NBR 7190, da ABNT (1997), e o modelo final proposto por essa norma. Ou seja, foram avaliados os comportamentos das seguintes variáveis:

$\mathrm{x}_{1}$ - menor resultado obtido entre as 12 pranchas, em cada inclinação;

$\mathrm{x}_{\mathrm{wm}}$ - média dos resultados obtidos nas 12 pranchas, em cada inclinação;

$\mathrm{z}_{\mathrm{b}}$ - operador definido por:

$$
\mathrm{z}_{\mathrm{b}}=\left(2 \frac{\mathrm{x}_{1}+\mathrm{x}_{2}+\ldots+\mathrm{x}_{\frac{\mathrm{n}}{2}-1}}{\frac{\mathrm{n}}{2}-1}-\mathrm{x}_{\frac{\mathrm{n}}{2}}\right)
$$

Onde os $x_{i}$ representam os resultados, ordenados crescentemente, obtidos nas 12 pranchas, em cada inclinação;

Valor característico definido na NBR 7190, da ABNT (1997) - obtido por:

$$
\text { Máximo }\left\{\mathrm{x}_{1} ; 0,7 \cdot \mathrm{x}_{\mathrm{wm}} \text { e } \mathrm{x}_{\mathrm{wk}}=\left(2 \frac{\mathrm{x}_{1}+\mathrm{x}_{2}+\ldots+\mathrm{x}_{\mathrm{n}}-1}{\frac{\mathrm{n}}{2}-1}-\mathrm{x}_{\frac{\mathrm{n}}{2}}\right) 1,1\right\}
$$

Onde os $\mathrm{x}_{\mathrm{i}}$ representam os resultados, ordenados crescentemente, obtidos nas 12 pranchas, em cada inclinação.

Ressalta-se que, na realidade, foi estudada a variação da resistência característica ao cisalhamento com o ângulo entre o esforço aplicado e a direção das fibras, cujo comportamento é o mesmo, apenas com alteração de escala, ao da correspondente resistência de cálculo. Foram utilizados os modelos apresentados nas Equações 1 a 4.

Para a análise estatística, de cada prancha (ou variável utilizada na avaliação das resistências 
de cálculo), preparou-se uma planilha eletrônica, no aplicativo Microsoft Excel, na qual os resultados foram pareados aos valores calculados com os modelos, onde o expoente " $n$ " podia ser alterado. Destes pares de valores foram obtidos os desvios $\left(\mathrm{d}=\mathrm{f}_{\text {experimental }}-\mathrm{f}_{\text {modelo }}\right)$, o correspondente intervalo de confiança da média (Equação 9) e o diagrama dos desvios $(\mathrm{d} x \alpha)$. O valor do expoente " $n$ " foi sistematicamente alterado, com incrementos de 0,01 , até obter o intervalo em que o valor de " $n$ " atendesse, simultaneamente, o teste de pairing (intervalo de confiança da média contendo o zero) e o teste dos desvios (desvios não apresentando tendenciosidade ao logo do domínio).

Os modelos que apresentaram um intervalo de " $n$ " aceitável em todas as pranchas podem ser utilizados para representar a variação da resistência em questão, com o ângulo entre o esforço aplicado e a direção das fibras da madeira, com qualquer valor de "n" do referido intervalo. Nesta avaliação, o erro estatístico pode ser minimizado retirando-se os valores destoantes de "n" (out limits).

\section{RESULTADOS E DISCUSSÃO}

Dos ensaios de cisalhamento, 11 tiveram de ser refeitos. A ruptura frágil, muito suscetível a defeitoslocalizados(nãopercebidos), provavelmente foi a responsável por este razoável número de resultados anômalos. Quanto ao modo de ruptura, todos os corpos de prova tiveram comportamento semelhante ao observado no cisalhamento paralelo às fibras.

Os ensaios de cisalhamento forneceram os valores das resistências (tensões de ruptura) ao cisalhamento inclinado, já corrigidos para o teor de umidade de 12\%, apresentadas na Tabela 1.

Aplicando o teste de pairing e o teste dos desvios, na comparação dos resultados dos ensaios (Tabela 1) aos modelos apresentados nas Equações 1 a 4, foram obtidos, para cada prancha, os limites do intervalo do expoente " $n$ " com validade estatística, bem como o valor do expoente " $n$ " que produz o melhor ajuste de cada modelo.

Ao se tentar obter um intervalo de validade comum, de cada modelo, nas várias pranchas, foram encontrados alguns intervalos nos quais o limite inferior é maior que o limite superior. Essas ocorrências foram classificadas como resultados destoantes (out limits).

Retirando-se os resultados destoantes (out limits), no máximo os dois resultados maiores e os dois menores, obtiveram-se resultados que indicam, para a madeira de peroba-mica, Aspidosperma populifolium A. DC., o seguinte: 1) A validade da Expressão de Hankinson (Equação 1), desde que o expoente " $n$ " esteja no intervalo $1,88 \leq n \leq 2,09$. O expoente que fornece melhor ajuste é $\mathrm{n}=2,05$. O valor tradicionalmente utilizado, de $n=2$, também apresentou validade estatística. 2) A validade da Expressão dos senos (Equação 3), desde que o expoente " $\mathrm{n}$ " esteja no intervalo $1,16 \leq \mathrm{n} \leq 1,27$. O expoente que fornece melhor ajuste é $\mathrm{n}=1,21$. O valor tradicionalmente utilizado pela norma alemã

TABELA 1: Resistências obtidas nos ensaios de cisalhamento de cada prancha.

TABLE 1: Strengths that were obtained in the shear tests of each board.

\begin{tabular}{|c|c|c|c|c|c|c|c|c|c|c|c|}
\hline \multirow{2}{*}{  } & \multicolumn{11}{|c|}{$\begin{array}{l}\text { Valores das resistências (tensão de ruptura) ao cisalhamento inclinado, } \\
\qquad f_{v, \alpha}(\mathrm{MPa}) \text {, nas seguintes inclinações } \alpha \text { : }\end{array}$} \\
\hline & $0^{\circ}$ & $10^{\circ}$ & $20^{\circ}$ & $30^{\circ}$ & $40^{\circ}$ & $45^{\circ}$ & $50^{\circ}$ & $60^{\circ}$ & $70^{\circ}$ & $80^{\circ}$ & $90^{\circ}$ \\
\hline A1 & 17,46 & 16,39 & 14,10 & 12,02 & 10,19 & 9,45 & 8,73 & 7,71 & 6,68 & 6,56 & 6,55 \\
\hline A2 & 17,03 & 16,31 & 15,06 & 13,18 & 11,13 & 10,11 & 9,09 & 7,83 & 6,56 & 6,27 & 5,84 \\
\hline B1 & 15,67 & 15,24 & 13,67 & 12,20 & 10,45 & 9,75 & 9,21 & 8,31 & 7,98 & 7,84 & 7,70 \\
\hline B2 & 14,98 & 15,04 & 14,16 & 13,54 & 11,79 & 11,26 & 9,74 & 7,72 & 6,82 & 6,60 & 6,49 \\
\hline $\mathrm{C} 1$ & 14,31 & 14,55 & 14,09 & 13,40 & 10,86 & 10,19 & 9,53 & 7,29 & 6,72 & 5,29 & 6,12 \\
\hline $\mathrm{C} 2$ & 14,87 & 14,04 & 13,28 & 10,84 & 8,54 & 7,89 & 7,24 & 6,35 & 5,90 & 5,81 & 5,78 \\
\hline D1 & 13,44 & 13,30 & 12,67 & 12,15 & 10,05 & 9,09 & 8,13 & 6,90 & 6,53 & 6,46 & 6,43 \\
\hline D2 & 14,49 & 14,49 & 13,62 & 12,10 & 10,04 & 9,25 & 8,46 & 7,35 & 6,47 & 6,22 & 6,13 \\
\hline E1 & 16,82 & 16,16 & 14,87 & 11,99 & 10,09 & 8,94 & 8,31 & 7,48 & 6,87 & 6,82 & 6,59 \\
\hline E2 & 15,28 & 14,16 & 12,28 & 10,77 & 9,57 & 8,85 & 8,46 & 7,75 & 7,04 & 6,81 & 6,82 \\
\hline F1 & 17,64 & 16,80 & 14,05 & 10,92 & 8,62 & 8,31 & 8,00 & 7,24 & 7,17 & 7,11 & 6,77 \\
\hline F2 & 14,79 & 14,87 & 13,54 & 11,17 & 9,47 & 8,19 & 7,96 & 7,01 & 6,51 & 6,42 & 6,43 \\
\hline
\end{tabular}


DIN-1052, segundo Pfeil (1978), de $\mathrm{n}=1$, não tem validade estatística. Este modelo também não tem validade com $n=2$, da proposta de Szücs (1992). 3) A validade da Expressão apresentada por Karlsen (Equação 2), desde que o expoente "n" esteja no intervalo $1,71 \leq \mathrm{n} \leq 2,26$. O expoente que fornece melhor ajuste é $\mathrm{n}=2,13$. O valor tradicionalmente utilizado, recomendado por Karlsen et al. (1967), de $\mathrm{n}=3$, não tem validade estatística. 4) A Expressão de Keylwerth (Equação 4) tem validade estatística para " $n$ " no intervalo $1,88 \leq n \leq 2,22$. O expoente que fornece melhor ajuste é $\mathrm{n}=2,04$. $\mathrm{O}$ valor tradicionalmente utilizado, recomendado por Kollmann e Côté Jr. (1984), de n = 2, também tem validade estatística.

Para avaliar a aplicação dos modelos na resistência de cálculo, os valores apresentados na Tabela 1, foram ordenados, crescentemente, e foram calculadas as variáveis utilizadas para avaliar a variação das resistências de cálculo. Estes valores são apresentados na Tabela 2.

Aplicando o teste de pairing e o teste dos desvios, na comparação dos resultados obtidos na Tabela 2 aos modelos apresentados nas Equações 1 a 4 , foram obtidos, para cada variável avaliada, os limites do intervalo do expoente " $n$ " com validade estatística, bem como o valor do expoente " $n$ " que produz o melhor ajuste de cada modelo.

Embora não se tenha evidência de que as variáveis utilizadas para estimar a resistência característica tenham o mesmo comportamento, procurou-se obter um intervalo de validade comum, de cada modelo, para as várias variáveis avaliadas. Estes resultados, para a madeira de peroba-mica, Aspidosperma populifolium A. DC., indicam:

1) A validade da Expressão de Hankinson (Equação 1), desde que o expoente " $n$ " esteja no intervalo $1,96 \leq \mathrm{n} \leq 2,08$. O expoente que fornece melhor ajuste é $\mathrm{n}=2,03$. O valor tradicionalmente utilizado, de $\mathrm{n}=2$, também tem validade estatística.

2) A validade da Expressão dos senos (Equação 3), desde que o expoente "n" esteja no intervalo $0,90 \leq n \leq 1,39$. O expoente que fornece melhor ajuste é $\mathrm{n}=1,18$. O valor tradicionalmente utilizado pela norma alemã DIN-1052, segundo Pfeil (1978), de $\mathrm{n}=1$, também tem validade estatística. Entretanto, este modelo não apresentou validade com $n=2$, da proposta de Szücs (1992).

TABELA 2: Valores das variáveis utilizadas para avaliar a variação das resistências de cálculo ao cisalhamento.

TABLE 2: Values of the variables used to evaluate the variation of shear strength calculus.

\begin{tabular}{|c|c|c|c|c|}
\hline \multirow{2}{*}{ 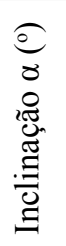 } & \multicolumn{4}{|c|}{$\begin{array}{l}\text { Valores das variáveis utilizadas para avaliar a variação das resistências de cálculo ao cisalhamento, em } \\
\text { MPa. }\end{array}$} \\
\hline & Valor mínimo $\left(\mathrm{f}_{\mathrm{v}, 1}\right)$ & Valor médio $\left(\mathrm{f}_{\mathrm{v}, \mathrm{m}}\right)$ & Operador $\mathrm{z}_{\mathrm{b}}{ }^{(1)}$ & Valor característico $\left(\mathrm{f}_{\mathrm{v}, \mathrm{k}}\right)^{(2)}$ \\
\hline $0^{\circ}$ & 13,44 & 15,56 & 13,78 & 15,16 \\
\hline $10^{\circ}$ & 13,30 & 15,11 & 13,35 & 14,68 \\
\hline $20^{\circ}$ & 12,28 & 13,78 & 12,49 & 13,74 \\
\hline $30^{\circ}$ & 10,77 & 12,02 & 10,26 & 11,28 \\
\hline $40^{\circ}$ & 8,54 & 10,07 & 8,44 & 9,29 \\
\hline $45^{\circ}$ & 7,89 & 9,27 & 7,78 & 8,55 \\
\hline $50^{\circ}$ & 7,24 & 8,57 & 7,40 & 8,14 \\
\hline $60^{\circ}$ & 6,35 & 7,41 & 6,56 & 7,22 \\
\hline $70^{\circ}$ & 5,90 & 6,77 & 6,11 & 6,72 \\
\hline $80^{\circ}$ & 5,29 & 6,52 & 5,53 & 6,09 \\
\hline $90^{\circ}$ & 5,78 & 6,47 & 5,69 & 6,25 \\
\hline
\end{tabular}

Em que: ${ }^{1} \mathrm{z}_{\mathrm{b}}=\left(2 \frac{\mathrm{f}_{\mathrm{v}, 1}+\mathrm{f}_{\mathrm{v}, 2}+\ldots+\mathrm{f}_{\mathrm{v}, \frac{\mathrm{n}}{2}-1}}{\frac{\mathrm{n}}{2}-1}-\mathrm{f}_{\mathrm{v}, \frac{\mathrm{n}}{2}}\right) \quad$ Máximo $\left\{\mathrm{f}_{\mathrm{v}, 1} ; 0,7 . \mathrm{f}_{\mathrm{v}, \mathrm{m}}\right.$ e $\mathrm{f}_{\mathrm{v}, \mathrm{k}}=\left(2 \frac{\left.\left.\mathrm{f}_{\mathrm{v}, 1}+\mathrm{f}_{\mathrm{v}, 2}+\ldots+\mathrm{f}_{\mathrm{v}, \frac{\mathrm{n}}{2}-1}-\mathrm{f}_{\mathrm{v}, \frac{\mathrm{n}}{2}}\right) 1,1\right\}}{\frac{\mathrm{n}}{2}-1}\right)$ 
3) A validade da Expressão apresentada por Karlsen (Equação 2), desde que o expoente "n" esteja no intervalo $1,88 \leq \mathrm{n} \leq 2,19$. O expoente que fornece melhor ajuste é $\mathrm{n}=2,04$. O valor tradicionalmenteutilizado, recomendado por Karlsen et al. (1967), de $n=3$, não tem validade estatística.

4) A Expressão de Keylwerth (Equação 4) tem validade estatística para "n" no intervalo 1,76 $\leq \mathrm{n} \leq 2,35$. O expoente que fornece melhor ajuste é $\mathrm{n}=2,16$. O valor tradicionalmente utilizado, recomendado por Kollmann e Côté Jr. (1984), de n $=2$, também tem validade estatística.

$\mathrm{Na}$ Figura 2 apresenta-se o ajuste dos modelos avaliados, ao estimador da resistência característica definido na NBR 7190, da ABNT (1997), usando o expoente tradicionalmente utilizado. Desta figura percebe-se que apenas dois modelos não são estatisticamente ajustados e que o melhor ajuste é obtido no modelo de Keylwerth (Equação 4).

$\mathrm{Na}$ Figura 3 apresenta-se o ajuste dos modelos avaliados, ao estimador da resistência característica definido na NBR 7190, da ABNT (1997), usando o melhor expoente para cada modelo. Desta figura percebe-se que todos os modelos podem ser estatisticamente ajustados, desde que se utilize o expoente adequado.

Para avaliar a sensibilidade das expressões à variação do expoente "n", analisou-se o erro máximo cometido na avaliação da resistência. Com o melhor expoente, as expressões de Hankinson (Equação 1), Karlsen (Equação 2) e Keylwerth (Equação 4), apresentaram erro máximo da ordem de $5 \%$, e a expressão dos Senos (Equação 3) de 8\%. Uma variação de 0,5 no valor deste expoente, nas expressões de Hankinson e dos Senos, elevou o erro

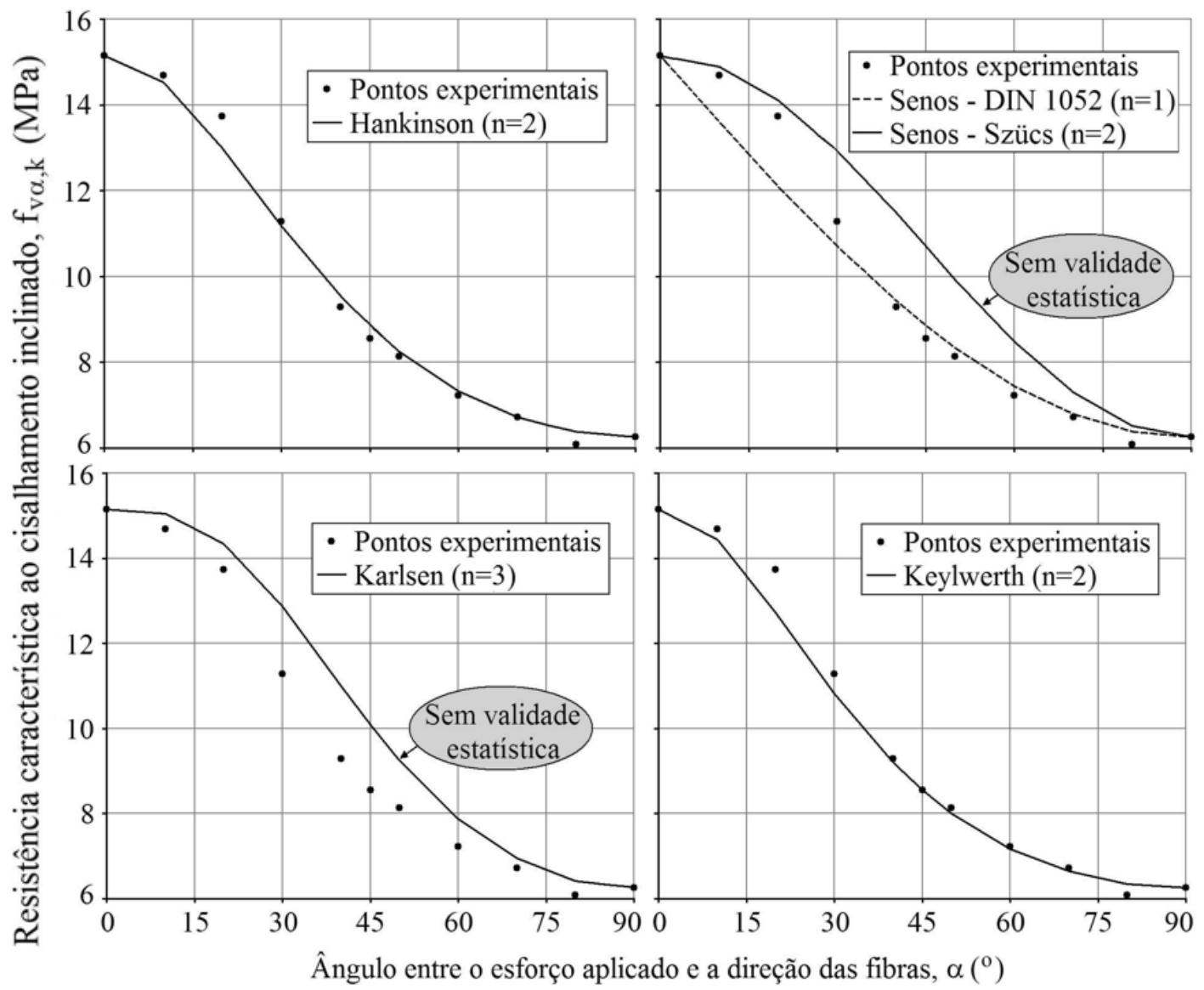

FIGURA 2: Ajuste dos diversos modelos, com os expoentes tradicionalmente utilizados, aos pontos experimentais, que correspondem à resistência característica ao cisalhamento obtida com o estimador proposto pela NBR 7190, da ABNT (1997).

FIGURE 2: Adjust of the several models, with the exponents traditionally used, to the experimental points that correspond to the characteristic shear strength obtained with the estimation proposed by NBR 7190, of ABNT (1997). 


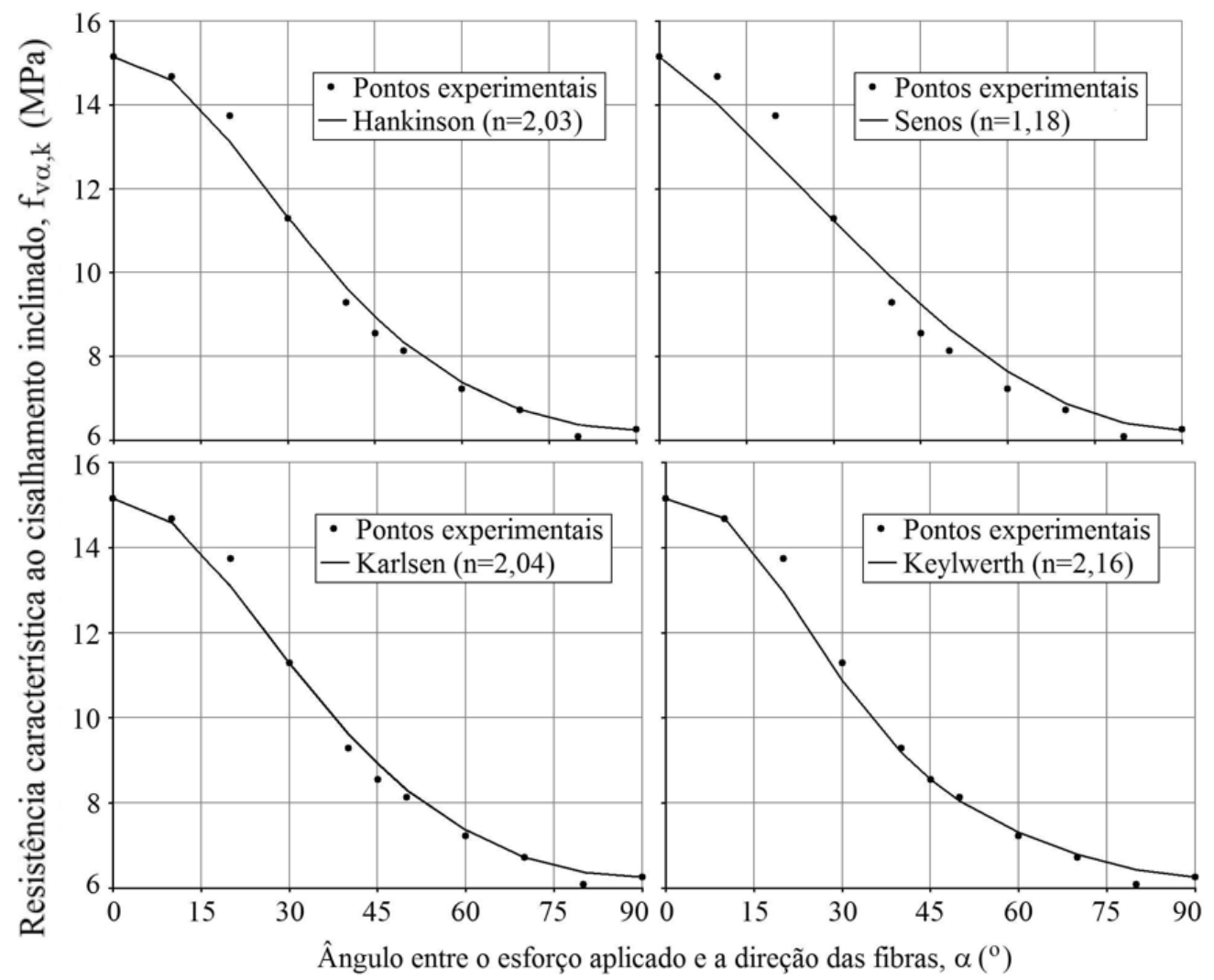

FIGURA 3: Ajuste dos diversos modelos, com os expoentes que fornecem melhores ajustes, aos pontos experimentais correspondentes à resistência característica ao cisalhamento obtida com o estimador proposto pela NBR 7190, da ABNT (1997).

FIGURE 3: Adjust of the several models, with the exponents that supply better fittings, to the experimental points, that correspond to the shear characteristic strength obtained with the estimation proposed by NBR 7190, of ABNT (1997).

máximo cometido na avaliação da resistência para aproximadamente $20 \%$, já na expressão de Karlsen o erro máximo chegou a $13 \%$ e na de Keylwerth não ultrapassou os $10 \%$. A variação unitária, em relação ao melhor expoente, elevou o erro cometido na avaliação da resistência para, aproximadamente, $35 \%$ com as expressões de Hankinson e dos Senos, $20 \%$ com a expressão de Karlsen e $13 \%$ com a expressão de Keylwerth.

O modelo de Keylwerth (Equação 4) foi o que apresentou melhor ajuste, seguido dos modelos de Hankinson (Equação 1) e de Karlsen (Equação 2). Deve-se ressaltar que, nos modelos de Hankinson e de Karlsen, pequenas variações no expoente alteram substancialmente o ajuste, já o modelo de Keylwerth é menos sensível a estas variações.

\section{CONCLUSÕES}

Esta pesquisa foi realizada com uma única espécie, assim, as conclusões apresentadas a seguir têm validade para essa espécie. Recomendam-se trabalhos semelhantes com outras espécies para poder generalizar essas conclusões.

Dos modelos encontrados na literatura (Equações 1 a 4), os modelos de Karlsen (Equação 2) e dos Senos, como proposto por Szücs (1992), não se mostraram estatisticamente válidos em seu formato original, tanto para explicar o comportamento individual, o médio, ou da porção mais fraca do material (valor característico). O modelo de Keylwerth (Equação 4) foi o que forneceu melhor ajuste.

A utilização da Expressão de Keylwerth (Equação 4) é interessante quando for feita a caracterização completa de resistência da madeira, entretanto, serão necessários os ensaios de cisalhamento perpendicular (rolling shear) e com inclinação de $45^{\circ}$, não previstos na atual NBR 7190 da ABNT (1997). 
Para caracterização mínima ou simplificada da madeira pode-se utilizar um modelo baseado em Keylwerth (Equação 4), mas utilizando valores fixos para as relações $f_{v 0} / f_{v 90}$ e $f_{v 0} / f_{v 45}$. Neste caso, será necessário definir em norma estes valores, que, para a peroba-mica, Aspidosperma populifolium A. DC., apresentaram os seguintes valores $\mathrm{f}_{\mathrm{v} 0} / \mathrm{f}_{\mathrm{v} 90}=2,4$ e $\mathrm{f}_{\mathrm{v} 0} / \mathrm{f}_{\mathrm{v} 45}=1,7$.

Os outros modelos avaliados, especialmente os de Hankinson (Equação 1) e Karlsen (Equação 2), com o expoente adequado, também fornecem bom ajuste. Entretanto, estes modelos são muito sensíveis à alteração do expoente, portanto, obter um expoente aceitável para todas as espécies (ou mesmo tipo de solicitação) pode ser tarefa impraticável. O modelo de Keylwerth mostrou-se mais tolerante à variação do expoente.

\section{AGRADECIMENTOS}

Os autores agradecem ao Conselho Nacional de Desenvolvimento Científico e Tecnológico $\mathrm{CNPq}$, pelo financiamento do projeto de pesquisa que deu origem a este trabalho.

\section{REFERÊNCIAS BIBLIOGRÁFICAS}

ASSOCIAÇÃO BRASILEIRA DE NORMAS TÉCNICAS. NBR 7190 - Projeto de estruturas de Madeira. Rio de Janeiro: ABNT, 1997. 107 p
KARLSEN, G. G. et al. Wooden Structures. Moscow: Mir, 1967. $638 \mathrm{p}$

KOLLMANN, F. F. P.; CÔTÉ JR., W. A. Principles of wood science and technology, vol. I Solid Wood. New York: Springer-Verlag, 1984, 502 p.

LOGSDON, N. B. Influência da umidade nas propriedades de resistência e rigidez da madeira. 1998. 174 f. Tese (Doutorado em Engenharia de Estruturas) - Escola de Engenharia de São Carlos - Universidade de São Paulo, São Carlos, 1998.

NICOLAS, A. N.; MASCIA, N. T.; TODESCHINI, R. Comparação entre o critério de Tsai-Wu e a fórmula de Hankinson na tração da madeira. Madeira: Arquitetura e Engenharia, São Carlos, v. 9, n. 23. p. 13-29, jul./dez. 2008.

PFEIL, W. Estruturas de madeira: dimensionamento segundo as normas brasileiras NB-11 e os modernos critérios das normas alemãs e americanas, Rio de Janeiro: Livros Técnicos e Científicos, 1978. $253 \mathrm{p}$

SZÜCS, C. A. Estudo do comportamento da madeira a esforços inclinados, In: ENCONTRO BRASILEIRO EM MADEIRAS E EM ESTRUTURAS DE MADEIRA, 4., 1992, São Carlos. Anais... São Carlos: Instituto Brasileiro das Madeiras e das Estruturas de Madeira, 1992. v. 2, p. 53-60.

TSAI, S. W.; WU, E. M. A general theory of strength for anisotropic materials. Journal of Composite Materials, n. 1, v. 5, p. 58-80. 1971. 